\title{
Analysis of Wind Ramping Product Formulations in a Ramp-constrained Power Grid
}

\author{
Kwami Senam A. Sedzro \\ National Renewable Energy \\ Laboratory \\ Kwami.Sedzro@nrel.gov
}

\author{
Xin Fang \\ National Renewable Energy \\ Laboratory \\ Xin.Fang@nrel.gov
}

\author{
Bri-Mathias Hodge \\ National Renewable Energy \\ Laboratory \\ Bri-Mathias.Hodge@nrel.gov
}

\begin{abstract}
Flexible ramping products are designed to compensate the variability and uncertainty of load and intermittent generation. Since their market implementation by the California Independent System Operator (CAISO) and Midcontinent System Operator (MISO), flexible ramping products have garnered much attention. However, it is still unclear how to best formulate wind power plants' participation in the ramping requirement. This paper investigates different wind ramping product formulations and increasing wind power penetration in the context of a security-constrained unit commitment (SCUC) model. We demonstrate that the ramping model that captures both the intra-and inter-temporal output ramp capability of individual wind power plants reflects the true ramp contribution of the wind fleet. With increasing wind penetration, wind generation curtailments can support the grid's ramping needs. In addition, we found that increased wind penetration has the potential of lowering ramping and production costs. Numerical case studies performed on the TAMU 2000bus synthetic network support the findings.
\end{abstract}

\section{Nomenclature}

\subsection{Sets}

$B_{L} \quad$ Set of load buses

$B_{G} \quad$ Set of load buses

G Set of non-wind generation units

$L \quad$ Set of transmission lines

$T \quad$ Set of time slots for unit commitment problem

$\Omega \quad$ Set wind power plants

\subsection{Indices}

$b \quad$ Index for loads/load buses

$g \quad$ Index for non-wind generation units

$i \quad$ Index for generation buses

$l \quad$ Index for transmission lines $t \quad$ Index for time intervals

$w \quad$ Index for wind power plants

\subsection{Constants}

$C_{g}^{k} \quad$ Marginal cost of generation for unit $g$ in its block $k$

$D_{b, t} \quad$ Demand of bus b at time t

$D_{t} \quad$ System level demand at time $\mathrm{t}$

$D T_{g} \quad$ Minimum down time (MDT) for unit $g$

$\Delta P_{g}^{k} \quad$ Generation block size of unit $g$ 's block $k$

$G S F_{l-i}$ Generation shift factor from bus $i$ to line $l$

$I D T_{g} \quad$ Initial minimum down time (IMDT) for unit $g$

$I U T_{g} \quad$ Initial minimum up time (IMUT) for unit $g$

$K_{g} \quad$ Number of blocks in the cost function of generation unit $g$

Limit $_{l}$ Transmission limit for line $l$

$L S P_{b} \quad$ Load-shedding penalty factor for bus $b$

$N L_{g} \quad$ No-load cost of generation unit $g$

$\bar{P}_{w, t} \quad$ Forecasted wind power (upper bound) for plant $w$ at time $t$

$P_{g}^{\max } \quad$ Maximum generation output for unit $g$

$P_{g}^{\min } \quad$ Minimum generation output for unit $g$

$R_{g}^{U} \quad$ Ramp-up limit for unit $g$

$R_{g}^{D} \quad$ Ramp-down limit for unit $g$

$R_{g}^{S U} \quad$ Ramp-up limit for unit $g$ at the start-up stage

$R_{g}^{S D} \quad$ Ramp-down limit for unit $g$ at the shutdown

$R D_{g} \quad$ Ramp-down cost of unit $g$

$R R R_{t}^{U} \quad$ Regulating up reserve requirement of the system at time $t$

$R U_{g} \quad$ Ramp-up cost of unit $g$

$S D_{g} \quad$ Shutdown cost of unit $g$

$S R R_{t} \quad$ Spinning reserve requirement of the system at time $t$

$S U_{g} \quad$ Start-up cost of unit $g$

$U T_{g} \quad$ Minimum up time (MUT) for unit $g$

\subsection{Variables}

$\delta_{b, t} \quad$ Load-shedding quantity of bus $b$ at time $t$ 
$f r d_{g, t} \quad$ Flexible ramp-down capacity provided by unit $g$ at time interval $t$

$f r d_{w, t} \quad$ Flexible ramp- down capacity provided by wind power plant $w$ at time interval $t$

$f r u_{g, t} \quad$ Flexible ramp-up capacity provided by unit $g$ at time interval $t$

$f r u_{w, t} \quad$ Flexible ramp-up capacity provided by wind power plant $w$ at time interval $t$

$l s_{t} \quad$ System level load-shedding penalty at time $t$

$p_{g, t} \quad$ Generation output for unit $g$ at time interval $t$

$\bar{p}_{g, t} \quad$ Maximum available generation output for unit $g$ in time interval $t$

$p_{g, t}^{k} \quad$ Generation output of unit $g$ in its block k at time $t$

$p_{i, t}^{i n j} \quad$ Total generation injection at bus $i$ in time interval $t$

$p_{w, t} \quad$ Wind power output for unit w at time $t$

$p c_{g, t} \quad$ Production cost for unit $g$ at time $t$

$r c_{g, t} \quad$ Ramping cost of unit $g$ at time $t$

$r r_{g, t} \quad$ Regulating reserve capacity of unit $g$ at time $t$

$s d_{g, t} \quad$ Shutdown cost of unit $g$ at time $t$

$s r_{g, t} \quad$ Operating reserve capacity of unit $g$

$s u_{g, t} \quad$ Start-up cost of unit $g$ at time $t$

$v_{g, t} \quad$ Commitment status for unit $g$ at time $t$

\section{Introduction}

Recently, the substantially increasing integration of variable renewable energy sources, such as wind power, has significantly changed the generation portfolios of many electric power systems [1-4]. To operate power systems with high penetration levels of variable renewables, the systems need more flexible resources to mitigate the variability and uncertainty [5-10]. In realtime operations with high penetration of renewables, one of the major challenges is ensuring sufficient ramping capability. Therefore, they are usually modeled as "non-dispatchable" resources; a modeling approach that relies mostly on the existing system ramping capacities [11].

To deal with the potential ramping shortage issues that can arise from high renewable penetration levels, some independent system operators (ISOs) have launched and implemented market designs for flexible ramping products, such as the flexi-ramp product in the real-time market by the California Independent System Operator (CAISO) and the ramp capability product in the day-ahead and real-time markets by the Midcontinent Independent System Operator (MISO) $[6,7]$. In these designs, specifically in the real-time markets, flexible ramp capacity is procured by adding ramp requirements in the original deterministic realtime economic dispatch (RTED) models [8], such that the ramp product is co-optimized with energy and other ancillary services in the real-time energy market. In these market designs, wind power is usually treated as a source of uncertainty that contributes to the ramping requirement in the system; however, the use of wind power to increase system reliability and flexibilityincluding modeling it as a flexible ramp capacity provider - is attracting more attention due to the rapidly increasing penetration of wind power [9-11]. The system flexible ramp requirements and the impacts of ramp products on market clearing have been analyzed in [12-16]. Reference [17] studies the potential for wind power to provide flexible ramping products in the realtime market as an additional value stream. In $[15,16]$, the impact of wind power on flexible ramping was reviewed. These studies analyze the impacts of flexible ramping on a system's reliability and its operation costs from the system operator standpoint. Further, the benefits of wind power providing ramp products were analyzed in $[5,9,18]$ to improve system reliability and reduce ramp scarcity. Reference [19] introduced the concept of flexible dispatch margin as an opportunity for wind resources to participate in reducing variability and uncertainty as renewable penetration increases. From a renewable power plant owner point of view, [20-22] investigate optimal energy and reserve offering strategies that maximize plant revenues while considering the risk of profit loss.

However, in the existing literature, not much attention has been devoted to the impact of flexible ramping product mathematical formulation. The general formulation considers the ramping product as intertemporal variation of power output. A direct application of this modeling approach to renewable based-ramping resources can lead to unnecessary curtailments. In addition, since the ramping products are procured to mitigate uncertainties and variabilities in the power system, it is critical to account for power forecasts when formulating the participation of non-dispatchable resources, such as wind power, in the flexible ramping market. We further study how the economic impact of wind ramping products scales across increasing wind penetration levels.

In this paper, the possibility of wind power to provide flexible ramping products is further analyzed. We propose three different models to incorporate the ramping products from wind power based on wind power forecasts. A case study is conducted on a large system with two thousand buses to analyze the impact of wind power ramping products modeling, as well as wind power penetration, on the system costs and ramping reserve capability.

The rest of this paper is organized as follows. Section 3 introduces different flexible ramping product formulations in the day-ahead SCUC model. Section 4 
evaluates the alternative ramping models through case studies in a synthetic two thousand bus system and analyzes the impact of wind power ramping products on the system operating cost and reserve capability. Section 5 concludes the paper.

\section{Unit commitment problem with flexible ramping products}

This section presents the overall formulation of the SCUC problem including flexible ramping products.

\subsection{Objective function}

The SCUC problem studied in this paper seeks to minimize the overall operating cost, made up of startup costs $\left(s u_{g, t}\right)$ and shutdown costs $\left(s d_{g, t}\right)$, production costs $\left(p c_{g, t}\right)$, ramping costs $\left(r c_{g, t}\right)$ and load shedding costs $\left(l s_{t}\right)$ costs as follows:

$$
\sum_{t \in T}\left[\sum_{g \in G} s u_{g, t}+s d_{g, t}+p c_{g, t}+r c_{g, t}\right]+l s_{t}
$$

with:

$$
\begin{gathered}
s u_{g, t} \geq S U_{g}\left[v_{g, t}-v_{g, t-1}\right], \forall g \in G, t \in T \\
s d_{g, t} \geq S D_{g}\left[v_{g, t-1}-v_{g, t}\right], \forall g \in G, t \in T \\
p c_{g, t}=N L_{g} v_{g, t}+\sum_{k=1}^{K_{g}} p_{g, t}^{k} C_{g}^{k}, \forall g \in G, t \in T \\
r c_{g, t}=R U_{g} f r u_{g, t}+R D_{g, t} f r d_{g, t}, \forall g \in G, t \in T \\
l s_{t}=\sum_{b \in B_{L}} L S P_{b} \delta_{b, t}, \forall t \in T
\end{gathered}
$$

where (1.a) and (1.b) define the unit startup cost and shutdown costs, respectively; (1.c) represents the generation production cost; and (1.d) is the system ramping product procurement costs. We use (1.e) to evaluate the impact of the distribution of load curtailment penalty. Equation (1.e) considers a locational curtailment penalty $L S P_{b}$. We consider block generator cost functions with the assumption that: $0 \leq C_{g}^{k}<C_{g}^{k+1}$, for all generator unit $g$ and block $k=$ $1, \ldots, K_{g}$.

\subsection{Constraints for a single unit}

We adopt similar constraints for traditional thermal units as in $[23,24]$, presented as follows for the sake of completeness:

$$
\begin{aligned}
& \sum_{t=1}^{I U T_{g}}\left(1-v_{g, t}\right)=0, \forall g \in G \\
& \sum_{\tau=t}^{t} v_{g, \tau} \geq U T_{g}\left(v_{g, t}-v_{g, t-1}\right), \forall g \in G, \\
& t=I U T_{g}+1, \ldots,|T|-U T_{g}+1 \\
& \sum_{\tau=t}^{T}\left[v_{g, \tau}-\left(v_{g, t}-v_{g, t-1}\right)\right] \geq 0, \forall g \in G \\
& t=|T|-U T_{g}+2, \ldots,|T| \\
& \sum_{t=1}^{I D T_{g}} v_{g, t}=0, \forall g \in G \\
& \sum_{\tau=t}^{t+D T_{g}-1}\left(1-v_{g, \tau}\right) \geq D T_{g}\left(v_{g, t-1}-v_{g, t}\right), \forall g \in G, \\
& t=I D T_{g}+1, \ldots,|T|-D T_{g}+1 \\
& \sum_{\tau=t}^{T}\left[1-v_{g, \tau}-\left(v_{g, t-1}-v_{g, t}\right)\right] \geq 0, \forall g \in G, \\
& t=|T|-D T_{g}+2, \ldots,|T| \\
& \bar{p}_{g, t}-p_{g, t-1}-r r_{g, t}^{U}-s r_{g, t} \\
& \leq R_{i}^{U} v_{g, t-1}+R_{i}^{S U}\left(v_{g, t}-v_{g, t-1}\right) \\
& +P_{g}^{\max }\left(1-v_{g, t}\right), \forall g \in G, t \in T \\
& \bar{p}_{g, t} \leq R_{g}^{S D}\left(v_{g, t}-v_{g, t+1}\right)+P_{g}^{\max } v_{g, t+1}, \forall g \in G, \\
& t=1, \ldots,|T|-1 \\
& p_{g, t}+s r_{g, t} \leq \bar{p}_{g, t}, \forall g \in G, t \in T \\
& p_{g, t-1}-p_{g, t} \leq R_{g}^{D} v_{g, t}+R_{g}^{S D}\left(v_{g, t-1}-v_{g, t}\right) \\
& +P_{g}^{\max }\left(1-v_{g, t-1}\right), \forall g \in G, t \in T \\
& p_{g, t}=P_{g}^{\min } v_{g, t}+\sum_{k=1}^{K_{g}} p_{g, t}^{k}, \forall g \in G, t \in T \\
& 0 \leq p_{g, t}^{k} \leq \Delta P_{g}^{k}, \forall g \in G, t \in T \\
& P_{g}^{\text {min }} v_{g, t} \leq p_{g, t} \leq \bar{p}_{g, t}, \forall g \in G, t \in T \\
& 0 \leq \bar{p}_{g, t} \leq P_{g}^{\max } v_{g, t}, \forall g \in G, t \in T \\
& p_{g, t}-f r d_{g, t} \geq P_{g}^{\text {min }} v_{g, t+1}, \forall g \in G, t=1, \ldots,|T|-1 \text { (6.a) } \\
& p_{g, t}+f r u_{g, t} \leq \bar{p}_{g, t+1}, \forall g \in G, t=1, \ldots,|T|-1
\end{aligned}
$$

where (2.a), (2.b) and (2.c) express the minimum uptime constraints and (3.a) through (3.c), the minimum downtime constraints of generation units. Equations (4.a) through (4.d) ensure that adequate reserve and ramping capability are available. Constraints (5.a) and (5.b) define the temporal output of each generation unit as an aggregate of its generation across all pricing blocks. Equations (5.c) and (5.d) express the feasible bounds of generation units' actual and available outputs. Constraints (6.a) and (6.b) bound flexible ramping-up and ramping-down products in the case of thermal generation units. 


\subsection{Wind power as flexible ramp provider}

The participation of wind power plants in the energy market as flexible ramp providers is modeled in the form of the following constraints:

$$
\begin{array}{r}
\left.p_{w, t}+f r u_{w, t} \leq \bar{P}_{w, t+1}, \forall w \in \Omega, t=1, \ldots,|T|-1 \quad \text { (7.a. } 1\right) \\
p_{w, t}+f r u_{w, t} \leq \bar{P}_{w, t}, \forall w \in \Omega, t=1, \ldots,|T| \quad \text { (7.a.2) } \\
p_{w, t}+f r u_{w, t} \leq \max \left\{\bar{P}_{w, t}, \bar{P}_{w, t+1}\right\}, \forall w \in \Omega, \\
t=1, \ldots,|T|-1 \\
p_{w, t}-f r d_{w, t} \geq 0, \forall w \in \Omega, t=1, \ldots,|T|
\end{array}
$$

Note that (7.a.1), (7.a.2) and (7.a.3) are exclusive and represent three different up-ramp formulations that are further discussed and tested in the case study section. According to (7.a.1), the flexible ramping-up product is defined solely in reference to the intertemporal difference between the current output and the available generation of the next time slot. The ramping product model (7.a.2), on the other hand, proposes an intratemporal approach, while (7.a.3) captures both the intertemporal and intra-temporal ramping opportunities.

\subsection{System constraints}

The system constraints include the energy balance constraint for every time interval, system operating reserve, flexible ramping, and transmission constraints, as follows:

$$
\begin{gathered}
\sum_{g \in G} p_{g, t}+\sum_{w \in \Omega} p_{w, t}-\sum_{b \in B_{L}} D_{b, t}-\delta_{b, t}=0, \forall t \in T \\
\delta_{b, t} \leq D_{b, t}, \forall b \in B_{L}, t \in T \\
\sum_{g \in G} s r_{g, t} \geq S R R_{t}, \forall t \in T \\
s r_{g, t} \leq R_{g}^{U}, \forall g \in G, t \in T \\
\sum_{g \in G} r r_{g, t}^{U} \geq R R R_{t}^{U}, \forall t \in T \\
\sum_{g \in G} r r_{g, t}^{D} \geq R R R_{t}^{D}, \forall t \in T \\
r r_{g, t}^{U} \leq R_{g}^{U}, \forall g \in G, t \in T \\
r r_{g, t}^{D} \leq R_{g}^{D}, \forall g \in G, t \in T \\
\sum_{i \in B_{G}} G S F_{l-i} p_{i, t}^{i n j}-\sum_{b \in B_{L}} G S F_{l-b}\left(D_{b, t}-\delta_{b, t}\right) \\
\leq L i m i t_{l}, \forall t \in T, l \in L
\end{gathered}
$$

where (8.a) is the energy balance constraint and (8.b), the load-shedding limit constraint. Constraints (9.e) and (9.f) enforce regulating reserve limits, while (9.c) and (9.d) ensure that the regulating reserve requirements are satisfied. Equation (9.b) imposes spinning reserve rate limits. Spinning reserve requirements are expressed by (9.a). Equation (10) enforces network flow constraints.

\section{Case studies}

The purpose of the study in this section is to test and evaluate the proposed alternative formulations described in Section 3.3. We also attempt to uncover possible impacts of wind penetration levels on rampconstrained power systems. We use the TAMU 2000bus synthetic grid test case as the basis of our analysis $[25,26]$ which is a simplified ERCOT power system with a moderate wind power penetration.

\subsection{The TAMU 2000-bus test system}

The 2000-bus synthetic case is "built from public information and a statistical analysis" [25] on the footprint of the state of Texas. With 2000 buses and 3206 branches, the case boasts a total generation capacity of 96,291.53 MW shared among coal, hydro, natural gas, nuclear, solar, and wind generating units as presented in Fig. 1.

At each bus, we use a nominal load profile weighted by the corresponding demand found in the 2000-bus power flow case data. Fig.2 shows the aggregate demand profile considered in this study.

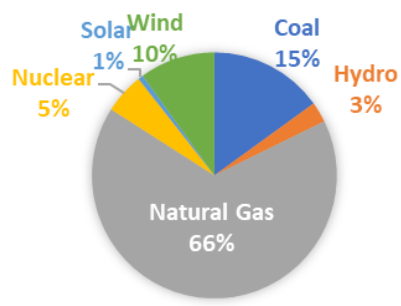

Figure 1. Generation mix

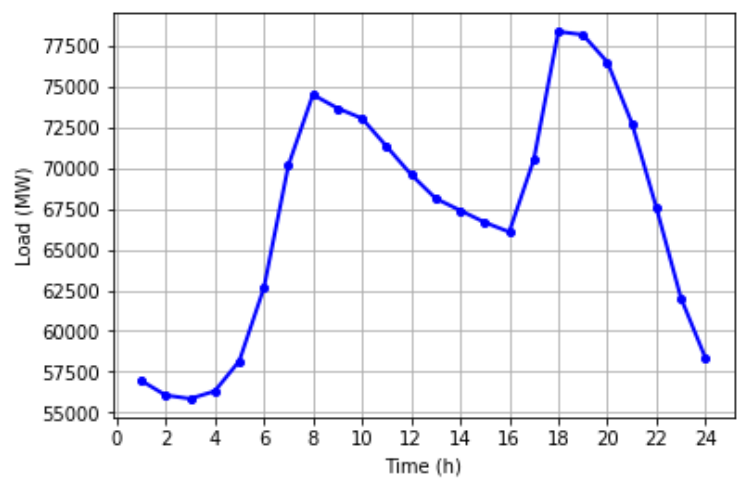

Figure 2. Aggregate load profile 
We assume that the ramp costs $\left(R U_{g}\right.$ and $\left.R D_{g}\right)$ for each generation unit are equal to $1 / 10^{\text {th }}$ of the marginal production cost. In order to create a more constrained system, we assume that the hydro and nuclear plants are offline.

\subsection{Impact of wind power ramping product formulation}

The wind ramping up product can be formulated three different ways as presented in Section 3.3, equations (7.a.1), (7.a.2) and (7.a.3). The study of the impact of each formulation is denoted by Case 1.a, Case 1.b, and Case 1.c respectively. For the sake of comparison, we study in Case 1base the base case where wind is not allowed to provide ramping products.

- Case 1.a: Intertemporal up-ramping model (7.a.1)

- Case 1.b: Intra-temporal up-ramping model (7.a.2)

- Case 1.c: Combined up-ramping model (7.a.3)

- Case 1base: Wind cannot provide ramping reserve (None of the 7.a equations)

The emphasis, in these case studies, is placed on the ramping-up product because the formulation of the ramping-down product (7.b) has not changed since curtailment of active power is easily performed in realtime and proactive curtailment is required to provide ramping-up product.

The choice of formulation is critical for wind ramping product valuation. Fig. 3 shows the impact of each of the three formulations on the actual dispatched wind output in a ramp-constrained system. It presents the aggregate available wind power and formulationspecific power output solutions.

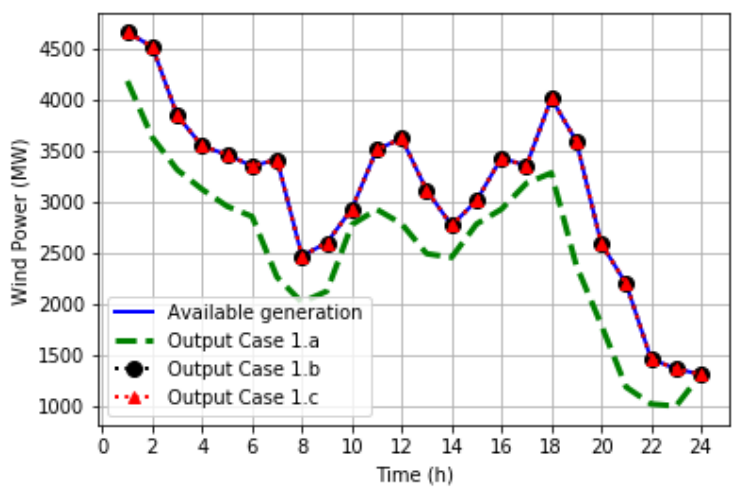

Figure 3. Aggregate wind power output profile for different ramping product formulations

In Case 1.b and Case 1.c, the outputs match exactly the available (forecasted) wind generation. In Case 1.a the wind power output at time $t, p_{w, t}$, is constrained to be less than the expected output in the next time interval $\bar{P}_{w, t+1}$, if there is a need or opportunity for an up-ramp product. The direct implication of this formulation is that whenever a wind ramping product is provided, wind generation is likely to be proactively curtailed in order to "secure" ramping capability.

Beyond the analysis of actual outputs, Fig.4 presents the aggregate ramping product available in each time interval according to each formulation.

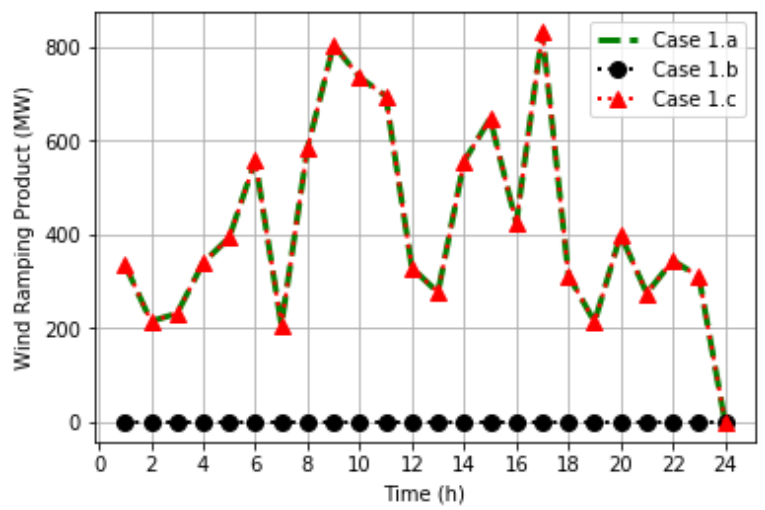

Figure 4. Aggregate wind ramping up product for different ramping product formulations

In Case 1.b, wind does not provide any ramping-up product. The formulation in Case 1.b defines the ramping product as the intra-interval ramping capability of units while Case 1.a defines it as an inter-interval ramping capability. Thus, wind is not able to provide any ramping capability in Case 1.b because the actual wind output is identical to the available wind generation (see Fig.3). Case 1.b could generate wind ramping-up product in the scenario where the ramping cost exceeds the energy cost. In that case, wind is curtailed.

Case 1.c, however, attempts to make the most out of both definitions. In fact, even though the wind power dispatch is the same as the available wind output as presented in Fig.3, the formulation in Case 1.c (see equation (7.a.3)) leverages both the natural intra- and inter-temporal ramping capabilities of individual wind power plants to provide ramping product as illustrated by Fig.5. During the intervals when the aggregated wind power is ramping down, some wind power plants still have the capability to provide the ramping-up product. Because the ramp directions are not uniform across all wind power plants, there is always a ramping-up potential even if the aggregate output decreases. This fact is formalized in the following lemma and proven below.

4.2.1. Lemma. For a non-empty and non-singleton set $\Omega$ of wind power plants operating across a time horizon of length at least greater than 1 , the following inequality holds: 


$$
\sum_{w \in \Omega} \max \left\{\bar{P}_{w, t}, \bar{P}_{w, t+1}\right\} \geq \max \left\{\sum_{w \in \Omega} \bar{P}_{w, t}, \sum_{w \in \Omega} \bar{P}_{w, t+1}\right\}
$$

\section{Definition: strict dominance}

$\bar{P}_{w, t}$ strictly dominates $\bar{P}_{w, t+1}$ on the set $\Omega$ of wind power plants if and only if, for all wind power plants $w \in \Omega, \bar{P}_{w, t}>\bar{P}_{w, t+1}$.

\subsubsection{Proof. Let's define:}

$$
\phi_{w, t}=\max \left\{\bar{P}_{w, t}, \bar{P}_{w, t+1}\right\}
$$

It is intuitive to write (12.b) from (12.a):

$$
\left\{\begin{array}{l}
\phi_{w, t} \geq \bar{P}_{w, t} \\
\& \\
\phi_{w, t} \geq \bar{P}_{w, t+1}
\end{array}\right.
$$

Given the spatial variability of the wind power generation, the following assumptions are valid:

- Assumption 1: Neither $\bar{P}_{w, t}$ nor $\bar{P}_{w, t+1}$ is strictly dominant, for all wind power plants $w$. The essence of this assumption is that the ramp direction is not the same for all wind power plants, from $t$ to $t+1$; some can ramp up while others ramp down. Given the complexity of atmospheric physics, this assumption is likely to hold for any non-trivial number of wind plants with realistic spatial separation at smaller time scales.

- Assumption 2: The aggregate wind generation is non-stationary, i.e.:

$$
\sum_{w} \bar{P}_{w, t} \neq \sum_{w} \bar{P}_{w, t+1}
$$

It follows from Assumptions 1 and 2 that (note the strict inequality):

$$
\left\{\begin{aligned}
\sum_{w} \phi_{w, t} & >\sum_{w} \bar{P}_{w, t} \\
\sum_{w} \phi_{w, t} & >\sum_{w} \bar{P}_{w, t+1}
\end{aligned}\right.
$$

We pose:

$$
\theta_{\mathrm{t}}=\max \left\{\sum_{w} \bar{P}_{w, t}, \sum_{w} \bar{P}_{w, t+1}\right\}
$$

Which is equivalent to:

$$
\theta_{\mathrm{t}}=\left\{\begin{array}{l}
\sum_{w} \bar{P}_{w, t}, \text { if } \sum_{w} \bar{P}_{w, t} \geq \sum_{w} \bar{P}_{w, t+1} \\
\sum_{w} \bar{P}_{w, t+1}, \quad \text { otherwise }
\end{array}\right.
$$

From (12.c) and (12.e), we have:

$$
\sum_{w} \phi_{w, t}>\theta_{\mathrm{t}}
$$

Using (12.a) and (12.d), we can rewrite (12.f) as:

$\sum_{w} \max \left\{\bar{P}_{w, t}, \bar{P}_{w, t+1}\right\}>\max \left\{\sum_{w} \bar{P}_{w, t}, \sum_{w} \bar{P}_{w, t+1}\right\}$

which partially completes the proof.

The equality in (11) is achieved in the special case of strict dominance (assumption 1 relaxed) or in case of stationarity of generation output (assumption 2 relaxed).

Equation (11) suggests that the total ramping product provided by wind on plant by plant basis exceeds the ramping capability of aggregate wind plant. The direct corollary of the lemma in equation (11) is that by formulating the wind ramping product as in equation (7.a.3), the system operator will account not only for the natural ramping but also for all necessary curtailments needed in providing the required ramp. The lemma is to highlights the system-wide effect of the formulation (7.a.3) applied on a plant by plant basis.

घ $-50.00-0.00=0.00-50.00=50.00-100.00=100.00-150.00$

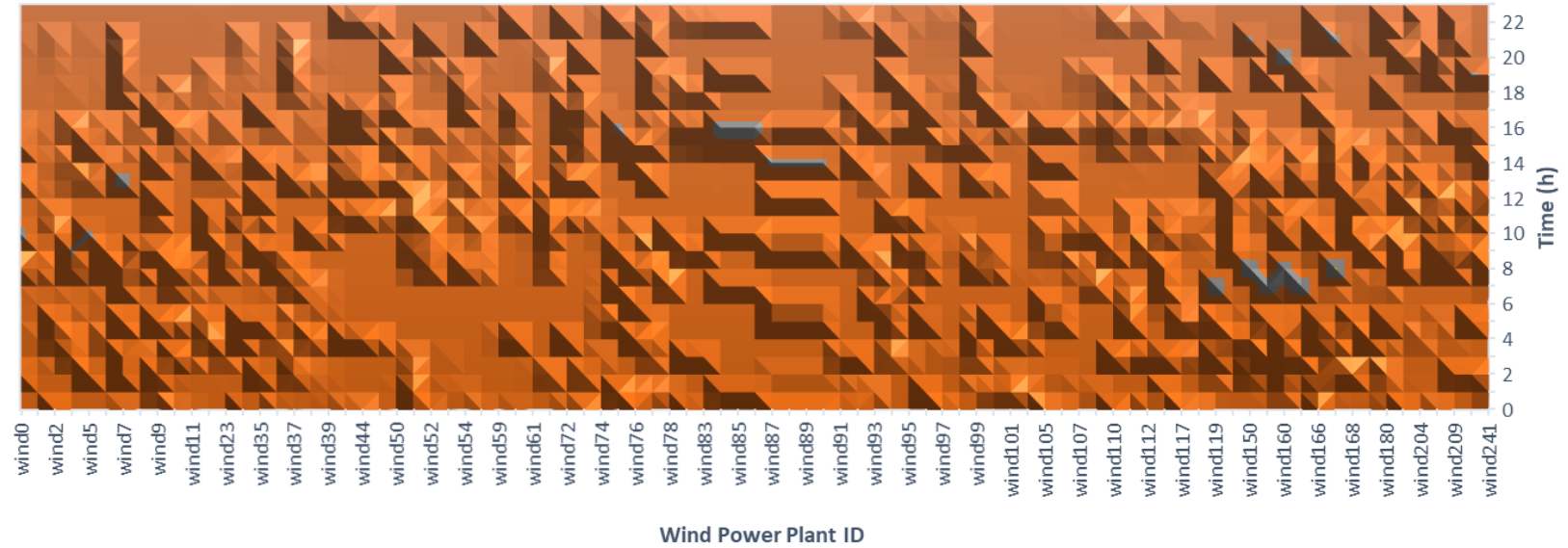

Figure 5. Up-ramp capability (MW) of individual wind power plants 
Table 1 presents the summary of the ramping and production costs obtained from the ramping formulations in Case 1a, Case $1 \mathrm{~b}$ and Case 1c. Caselbase corresponds to the base case in which wind is not allowed to provide any ramping product. In this case wind is not considered as a ramping resource. Thus, the security-constrained unit commitment model in Case1base does not include any wind ramping product. Conventional generators provide all necessary ramping products.

Table 1. Cost summary

\begin{tabular}{|l|l|c|}
\hline & Ramp. Cost (\$) & Prod. Cost (\$) \\
\hline Case 1base & 350,861 & $24,626,217$ \\
\hline Case 1.a & 282,322 & $24,794,378$ \\
\hline Case 1.b & 288,551 & $24,562,907$ \\
\hline Case 1.c & 271,430 & $24,545,787$ \\
\hline
\end{tabular}

In addition, Case 1a incurs a load curtailment cost of $\$ 116,390$ due to the wind power-shedding observed in Fig.3 (Output Case 1.a) while there is no curtailment in the other cases. Case 1.c outperforms all other cases in ramping as well as in production cost savings.

The analysis in this section reveals that the ramping product formulation in (7.a.3) is the one that captures the natural ramping capability of individual wind power plants without unnecessarily inducing wind curtailment that could be used to reduce production cost. It also accounts for and rewards any curtailment deemed necessary in the SCUC optimal solution.

The case study on the formulation impacts reveal that enabling wind power plants' participation in both energy and ramping markets, through appropriate modeling, can generate substantial operation cost savings. The next study investigates how the production cost drops with increasing wind penetration levels. For the rest of this paper, we use the ramping product formulation expressed in (7.a.3).

\subsection{Sensitivity to wind power penetration}

In this section, the study is centered on the impact of wind penetration on the production and ramping costs in a system where wind can provide ramping product.

We evaluate the ramping and production costs for different wind penetration levels from $10 \%$ (the baseline penetration) to $30 \%$. For each desired penetration level $\gamma^{\text {new }}$, the target wind plant capacity $P_{w}^{\max , n e w}$ is obtained by multiplying the initial wind capacity $P_{w}^{\text {max,init }}$ (i.e., the capacity at $10 \%$ wind penetration, in this case) of a wind power plant $w$ is multiplied by the scalar $\sigma$ given by:

$$
\sigma=\frac{\gamma^{\text {new }}}{\gamma^{\text {init }}} \times \frac{1-\gamma^{\text {init }}}{1-\gamma^{\text {new }}}
$$

For each penetration level, we compute the production and ramping costs via a 24-hour SCUC instance, with generation costs taken from the TAMU 2000 case data. Fig. 6 presents a quasi-linear cost reduction for wind penetration levels $10 \%$ through $30 \%$.

The additional wind generation displaces expensive thermal generation and provides energy up to available limits while contributing toward ramping needs using its natural ramping capability. This explains the ramping and production cost drop observed in Fig. 6

However, in ramp-constrained systems, wind can be curtailed in order to support the grid in providing the necessary ramp to the load. For instance, if between 3 to 8 am (see Fig.1), the load ramps up strongly but wind generation ramps down beyond the ramping-up capability of all other units and load combined, wind output will be shed as illustrated in Fig.7. Fig.7 shows the aggregate wind power output and available power at $28 \%$ wind penetration. In this particular case, wind is curtailed across all wind plants to support the up-ramp needs as demand rises between 1 and $6 \mathrm{am}$.

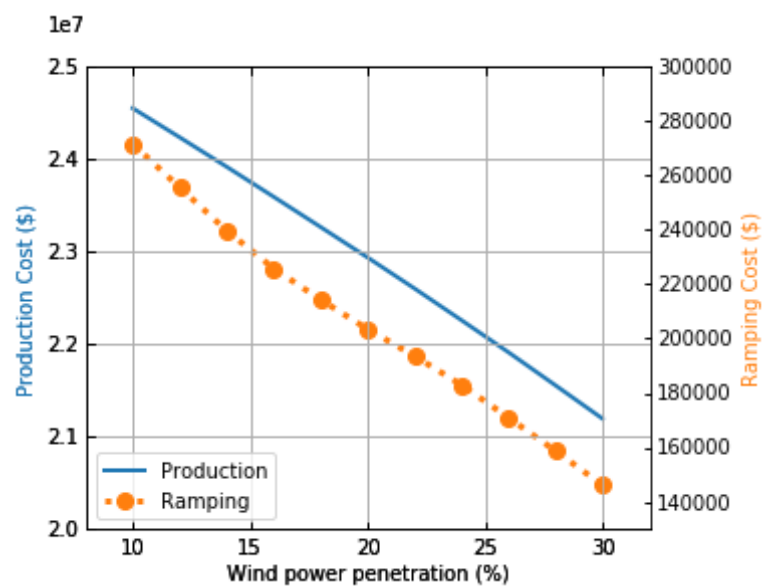

Figure 6. Sensitivity of production and ramping costs to wind power penetration

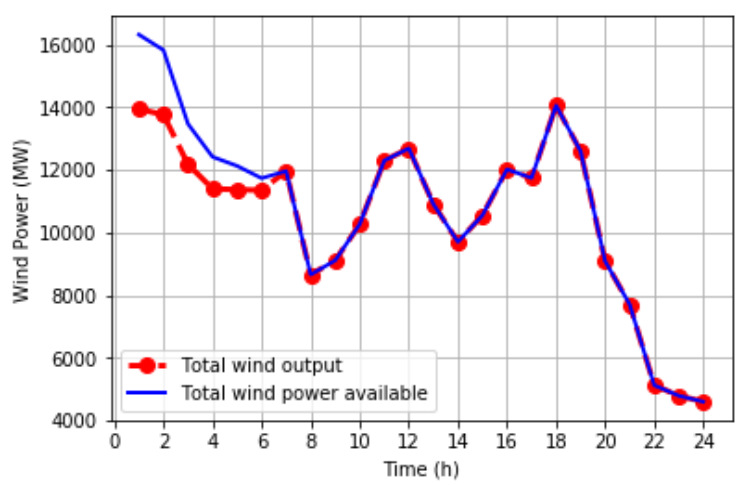

Figure 7. Wind curtailment for grid ramping support 
It is further noted that as wind penetration increases, the ramp contribution of wind power also increases. Fig. 8 shows the trend of wind contributions in providing for system-wide ramping-up needs. The primary y-axis on the left corresponds to the wind ramp contributions for $10 \%$ to $30 \%$ wind penetration. These contributions express the ratio between the available wind rampingup capability and the total ramping-up requirement.

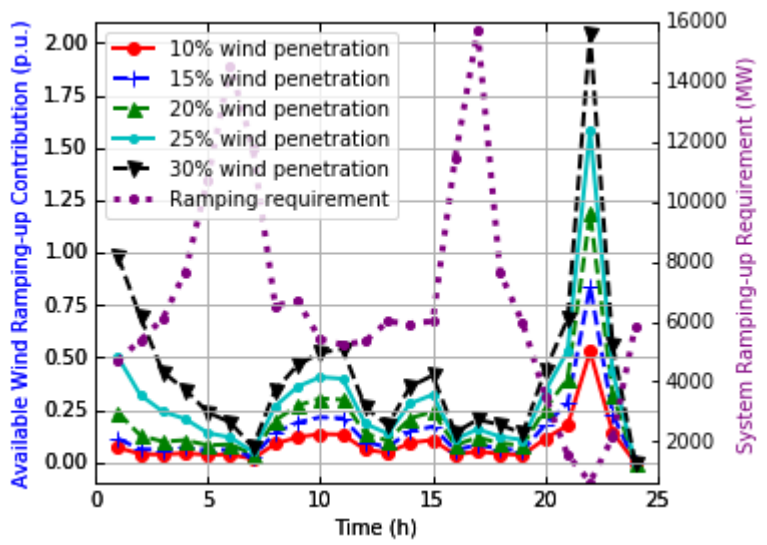

Figure 8. Wind ramping-up contribution

For the sake of reference, the secondary y-axis on the right indicates the system-wide ramping requirement. It is worth noting that the system ramping requirement depends solely on the system load, thus it does not change with wind penetration levels.

With increased wind penetration levels, both ramp and production costs decrease as wind resources displace more expensive generation units in providing both energy and ramping products. In cases where wind ramps in the opposite direction compared to the load, wind curtailment may occur in support to the grid.

\section{Conclusion}

This paper investigates three wind ramping-up product formulations, as well as the sensitivity of production and ramping costs with regards to increasing levels of wind penetration. The ramping product formulation in (7.a.3) captures the natural ramping capability of individual wind power plants without unnecessarily inducing wind curtailment that could be used to reduce production cost. As wind power penetration increases, the grid operating cost is expected to decrease even though wind might be curtailed to provide ramping support. This work presents deterministic study of the flexible ramping product formulation. Future work will focus on the robustness of the outperforming formulation in a stochastic setting where load and wind generation uncertainties are captured.

\section{Acknowledgements}

This work was authored by Alliance for Sustainable Energy, LLC, the Manager and Operator of the National Renewable Energy Laboratory for the U.S. Department of Energy (DOE) under Contract No. DE-AC3608GO28308. Funding provided by U.S. Department of Energy Office of Energy Efficiency and Renewable Energy Wind Energy Technologies Office. The views expressed in the article do not necessarily represent the views of the DOE or the U.S. Government. The U.S. Government retains and the publisher, by accepting the article for publication, acknowledges that the U.S. Government retains a nonexclusive, paid-up, irrevocable, worldwide license to publish or reproduce the published form of this work, or allow others to do so, for U.S. Government purposes.

\section{References}

[1] R. Chen, J. Wang, A. Botterud, and H. Sun, "Wind Power Providing Flexible Ramp Product," IEEE Transaction on Power Systems, vol. 32, no. 3, pp. 2049-2061, 2017.

[2] M. Cui, J. Zhang, H. Wu, and B. M. Hodge, "WindFriendly Flexible Ramping Product Design in MultiTimescale Power System Operations," IEEE

Transactions on Sustainable Energy, vol. 8, no. 3, pp. 1064-1075, 2017.

[3] M. Cui, D. Ke, Y. Sun, D. Gan, J. Zhang, and B.-M. Hodge, "Wind Power Ramp Event Forecasting Using a Stochastic Scenario Generation Method," Sustainable Energy, IEEE Transactions on, vol. 6, no. 2, pp. 422-433, 2015.

[4] H. Pandzic, Y. Dvorkin, T. Qiu, Y. Wang, and D. S. Kirschen, "Toward Cost-Efficient and Reliable Unit Commitment under Uncertainty," IEEE

Transactions on Power Systems, vol. 31, no. 2, pp. 970-982, 2016.

[5] B. Wang and B. F. Hobbs, "Real-Time Markets for Flexiramp: A Stochastic Unit Commitment-Based Analysis," IEEE Transactions on Power Systems, vol. 31, no. 2, pp. 846-860, 2016.

[6] E. Ela, and M. O. Malley, "Scheduling and Pricing for Expected Ramp Capability in Real-Time Power Markets," IEEE Transaction on Power Systems, pp. $1-11,2015$.

[7] N. Navid and G. Rosenwald, "Ramp Capability Product Design for MISO Markets," Mid-Continent System Operator, 2013.

[8] Q. Shi, F. Li, Q. Hu, and Z. Wang, "Dynamic demand control for system frequency regulation: Concept review, algorithm comparison, and future vision," Electric Power Systems Research, vol. 154, pp. 75-87, 2018.

[9] A. Nikoobakht and J. Aghaei, "IGDT-based robust optimal utilisation of wind power generation using coordinated flexibility resources," IET Renewable 
Power Generation, vol. 11, no. 2, pp. 264-277, 2016.

[10] California ISO, "Flexible Ramping Products: Revised Draft Final Proposal," pp. 1-18, 2015.

[11] R. Sevlian and R. Rajagopal, "Detection and statistics of wind power ramps," IEEE Transactions on Power Systems, vol. 28, no. 4, pp. 3610-3620, 2013.

[12] A. Cornelius, "Assessing the impact of flexible ramp capacity products in the Midcontinent ISO," Duke Univ., 2014.

[13] M. Cui, J. Zhang, C. Feng, A. R. Florita, Y. Sun, and B. M. Hodge, "Characterizing and analyzing ramping events in wind power, solar power, load, and netload," Renewable Energy, vol. 111, pp. 227244, 2017.

[14] C. Wu, G. Hug, S. Member, and S. Kar, "RiskLimiting Economic Dispatch for Electricity Markets With Flexible Ramping Products," IEEE

Transactions on Power Systems, pp. 1-14, 2015.

[15] B. Zhang and M. Kezunovic, "Impact on Power System Flexibility by Electric Vehicle Participation in Ramp Market," IEEE Transactions on Smart Grid, vol. 7, no. 3, pp. 1285-1294, 2016.

[16] Y. V. Makarov, C. Loutan, J. Ma, and P. de Mello, "Operational impacts of wind generation on California power systems," IEEE Transactions on Power Systems, vol. 24, no. 2, pp. 1039-1050, 2009.

[17] X. Fang, V. Krishnan, and B. Hodge, "Strategic Offering for Wind Power Producers Considering Energy and Flexible Ramping Products," Energies, vol. 11, no. 5, pp. 1239, 2018.

[18] E. Moiseeva, M. R. Hesamzadeh, and D. R. Biggar, "Exercise of Market Power on Ramp Rate in WindIntegrated Power Systems," IEEE Transactions on Power Systems, vol. 30, no. 3, pp. 1614-1623, 2015.

[19] J. B. Cardell, and C. L. Anderson, "A flexible dispatch margin for wind integration." IEEE
Transactions on Power Systems, vol. 30, no. 3, pp.1501-1510, 2015.

[20] K. S. Sedzro, S. Kishore, A. J. Lamadrid, and L. F Zuluaga, "Stochastic risk-sensitive market integration for renewable energy: Application to ocean wave power plants," Applied Energy, vol. 229, pp. 474-481, 2018.

[21] T. Soares, P. Pinson, T. V. Jensen, and H. Morais, "Optimal offering strategies for wind power in energy and primary reserve markets," IEEE Trans. Sustainable Energy 7.3, pp. 1036-1045, 2016.

[22] M. Hedayati-Mehdiabadi, J. Zhang, and K. W. Hedman, "Wind power dispatch margin for flexible energy and reserve scheduling with increased wind generation," IEEE Transactions on Sustainable Energy, vol. 6, no.4, pp 1543-1552, 2015.

[23] X. Fang, V. Krishnan, and B. Hodge, "Potential of Wind Power to Provide Flexible Ramping Products and Operating Reserve," in IEEE PES General Meeting, 2018, pp. 1-5.

[24] M. Carrion, J.M. Arroyo, and B. Hodge, "A Computationally Efficient Mixed-Integer Linear Formulation for the Thermal Unit Commitment Problem, " IEEE Transactions on Power Systems, vol. 21, no. 3, pp. 1371-1378, 2006.

[25] A.B. Birchfield, T. Xu, K.M. Gegner, K.S. Shetye, and T.J. Overbye, "Grid Structural Characteristics as Validation Criteria for Synthetic Networks, ” IEEE Transactions on Power Systems, vol. 32, no. 4, pp. 3258-3265, 2017.

[26] T. Xu , A.B. Birchfield, K.M. Gegner, K.S. Shetye, and T.J. Overbye, "Application of large-scale synthetic power system models for energy economic studies, "in Proceedings of the 50th Hawaii International Conference on System Sciences, 2017. 